\title{
Collapsibility of the Right Internal Jugular Vein Predicts Responsiveness to Fluid Administration in Patients Receiving Pressure Support Ventilation: A Prospective Cohort Study
}

\author{
Yusuke Iizuka $^{\mathrm{a}, \mathrm{e}}$, Takeshi Nomura ${ }^{\mathrm{b}, \mathrm{e}}$, Masamitsu Sanui ${ }^{\mathrm{a}, \mathrm{f}}$, Yasuhiro Mochida ${ }^{\mathrm{c}}$, \\ Akinori Aomatsu ${ }^{\text {a }}$, Alan Kawarai Lefor ${ }^{d}$
}

\begin{abstract}
Background: The aim of this study was to evaluate the utility of collapsibility of the internal jugular veins (IJVs) and subclavian veins (SCVs) in comparison with collapsibility of the inferior vena cava (IVC) in patients receiving pressure support ventilation.

Methods: Patients receiving pressure support ventilation were prospectively enrolled when fluid bolus administration was clinically indicated. The antero-posterior diameters of IJVs, SCVs and IVC were measured. Fluid responsiveness was defined as an $8 \%$ increase in stroke volume calculated with arterial pulse contour analysis after a passive leg raising maneuver.

Results: Twenty-seven patients (34 measurements) were included. Eighteen measurements were deemed fluid responsive. The area under the receiver operating characteristic curve of collapsibility of the right IJV antero-posterior diameter was 0.88 (95\% confidence interval (CI): $0.75-0.99$ ), while the area under the ROC curves for the antero-posterior diameter of the left IJV, right SCV, left SCV and the IVC were 0.57 (95\% CI: 0.37 - 0.77), 0.61 (95\% CI: 0.41 - 0.80), 0.55 (95\% CI: 0.35 - 0.76) and 0.57 (95\% CI: 0.37 - 0.77), respectively.
\end{abstract}

Conclusions: These results suggest that collapsibility of the right IJV

Manuscript submitted December 23, 2019, accepted February 19, 2020

${ }^{a}$ Department of Anesthesiology and Critical Care Medicine, Jichi Medical University Saitama Medical Center, 1-847 Amanuma, Omiya-ku, Saitatama city, Saitama 330-8503, Japan

bDepartment of Intensive Care Medicine, Tokyo Women's Medical University, 8-1, Kawada-cho, Shinjuku-ku, Tokyo, Japan

'Department of kidney disease and transplant center, Shonan Kamakura General Hospital, 1370-1 Okamoto, Kamakura city, Kanagawa 247-8533, Japan dDepartment of Surgery, Jichi Medical University, 3311-1 Yakushiji, Shimotsuke city, Tochigi 329-0498, Japan

'Yusuke Iizuka and Takeshi Nomura contributed equally as first authors.

${ }^{\mathrm{f} C}$ Corresponding Author: Masamitsu Sanui, Department of Anesthesiology and Critical Care Medicine, Jichi Medical University Saitama Medical Center, 1-847 Amanuma, Omiya-ku, Saitatama city, Saitama 330-8503, Japan.

Email: msanui@jichi.ac.jp

doi: https://doi.org/10.14740/jocmr4064 is a useful predictor of fluid responsiveness in patients receiving pressure support ventilation. Collapsibility of the IVC did not predict fluid responsiveness in these patients.

Keywords: Internal jugular vein; Subclavian vein; Inferior vena cava; Fluid responsiveness; Pressure support ventilation

\section{Introduction}

Fluid responsiveness, the ability of cardiac output to increase in response to a fluid infusion, is commonly used to evaluate intravascular fluid status in critically ill patients. For this purpose, dynamic parameters using mechanical ventilationinduced changes in preload, resulting in variation of stroke volume or pulse pressure (stroke volume variation (SVV) and pulse pressure variation, respectively) are far better predictors of fluid responsiveness than static parameters such as central venous pressure or pulmonary artery occlusion pressure $[1,2]$. However, the predictive values of these parameters in patients with spontaneous breathing, arrhythmias, low tidal volume ventilation and low lung compliance are suboptimal [3]. Collapsibility of the inferior vena cava (IVC) is a dynamic parameter with a relatively high ability to predict fluid responsiveness in patients receiving controlled mechanical ventilation [4, 5], but may not be a good predictor of fluid responsiveness in patients who are spontaneously breathing [6]. While many patients receive pressure support ventilation in the intensive care unit, the predictive ability of respiratory changes in the IVC to assess fluid responsiveness in these patients remains unknown.

Measurement of the IVC by echocardiography is simple, but adequate ultrasound images of the IVC are difficult to obtain in a substantial number of patients. Measurements of internal jugular veins (IJVs) and subclavian veins (SCVs) are easier to obtain [7], and the collapsibility of IJVs and SCVs may be useful as alternatives to the IVC. The ability of respiratory changes in the IJVs and SCVs to predict fluid responsiveness in patients on pressure support ventilation has not yet been evaluated.

We hypothesize that the collapsibility of IJVs and SCVs 


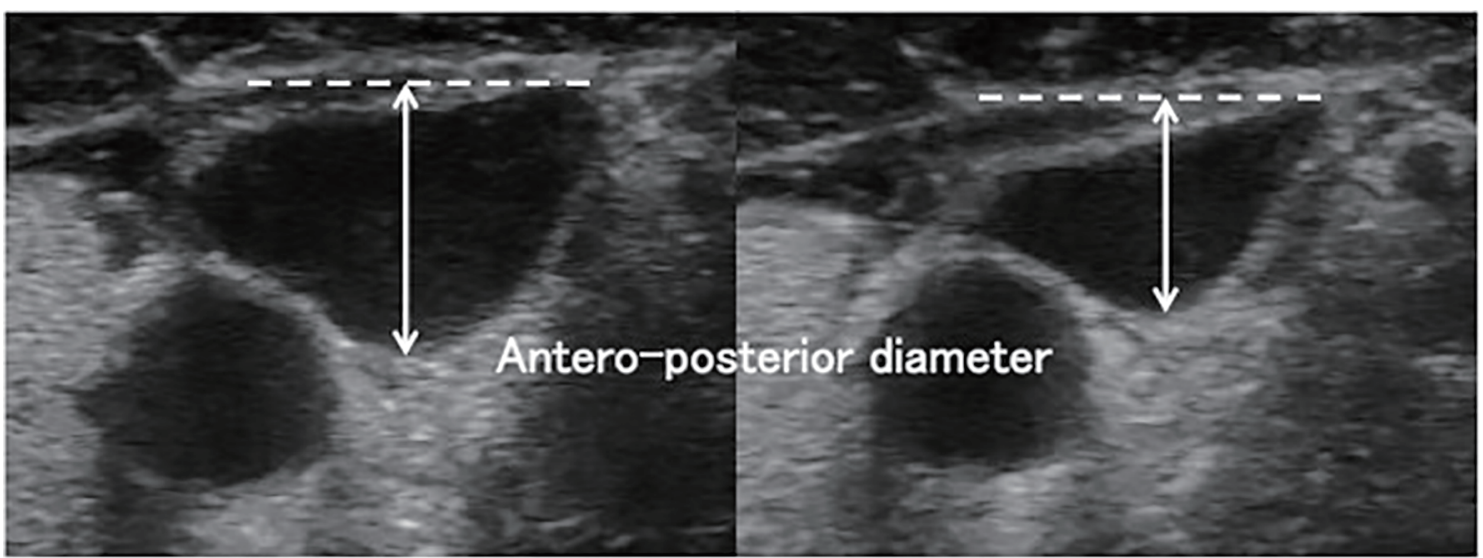

RIJV on expiration

RIJV on inspiration

Figure 1. Assessment of antero-posterior diameter of the right internal jugular vein by ultrasonography. The diameter was measured $2 \mathrm{~cm}$ above the level of the clavicle.

has a better area under the receiver operating characteristic (ROC) curve than collapsibility of the IVC with a reference to positive passive leg raising maneuver. The aim of this study was to compare the collapsibility of IJVs and SCVs with collapsibility of the IVC to predict fluid responsiveness in patients receiving pressure support ventilation.

\section{Materials and Methods}

\section{Study design}

This single-center, prospective observational study was performed in the surgical and medical intensive care unit of a tertiary hospital (Shonan Kamakura General Hospital, Kanagawa, Japan). Institutional Review Board approval was obtained (Shokama 20140327-1). This study was conducted in accordance with the Declaration of Helsinki. The study was registered in the University hospital Medical Information Network (UMIN000021449).

\section{Patients}

Study patients were: 1) intubated, mechanically ventilated receiving pressure support ventilation; 2) in acute circulatory failure and 3) who underwent placement of radial artery catheters, monitored by pulse contour analysis, Flotrac ${ }^{\mathrm{TM}}$ connected to arterial line and Vigileo ${ }^{\mathrm{TM}}$ monitors (version 3.06. Edwards, Irvine, CA, USA). Acute circulatory failure was defined as: 1) a systolic blood pressure less than $90 \mathrm{~mm} \mathrm{Hg}$ (or mean arterial pressure less than $65 \mathrm{~mm} \mathrm{Hg}$ ) or the need for vasopressors; 2) urine output below $0.5 \mathrm{~mL} / \mathrm{kg} / \mathrm{h}$ for at least $2 \mathrm{~h} ; 3$ ) tachycardia (heart rate $>100 \mathrm{bpm}$ ); or 4) the presence of skin mottling [8]. Patients with intracranial hypertension, abdominal compartment syndrome, pulmonary embolism, pulmonary hypertension, severe acute respiratory distress syndrome, positive end expiratory pressure (PEEP) higher than $10 \mathrm{~cm} \mathrm{H} 2 \mathrm{O}$, severe left ventricular dysfunction (ejection fraction less than $30 \%$ ), irregular heart rhythm, or occlusion of IJVs or SCVs or the IVC were excluded.

\section{Measurements}

For each patient, the following data were recorded: age (years), height $(\mathrm{cm})$, weight $(\mathrm{kg})$, diagnosis of acute circulatory failure, ventilator parameters $\left(\mathrm{FIO}_{2}\right.$, tidal volume $(\mathrm{mL})$, amount of pressure support $\left(\mathrm{cm} \mathrm{H}_{2} \mathrm{O}\right)$, respiratory rate, PEEP $\left(\mathrm{cm} \mathrm{H}_{2} \mathrm{O}\right)$, minute ventilation $(\mathrm{L} / \mathrm{min})$ ), hemodynamic parameters (heart rate (bpm), systolic/mean/diastolic arterial pressure $(\mathrm{mm} \mathrm{Hg})$, cardiac output $(\mathrm{L} / \mathrm{min})$, cardiac index $(\mathrm{L} / \mathrm{min} /$ body surface area), stroke volume (mL) and SVV (\%)). Echocardiography was performed (SSA-350A Corevision ${ }^{\mathrm{TM}}$, Toshiba, Japan) and the central veins were examined as follows: a linear probe was used to examine the IJVs and SCVs, and a sector probe was used to examine the IVC; antero-posterior diameter and cross-sectional area (CSA) of bilateral IJVs during a respiratory cycle were measured $2 \mathrm{~cm}$ above the level of the clavicle (Fig. 1). During the examination, the patient's chin was kept in the midline without neck extension. If a central venous catheter was in place in the examination area, we examined the area just upstream or downstream of insertion point as appropriate. While avoiding changes in venous diameter caused by probe compression, gentle pressure by the probe was applied to allow full collapse and expansion of the IJVs [7]. The SCVs were measured bilaterally at the point closest to where the SCV crosses the clavicle in a short axis view (Fig. 2). The IVC was measured using a subxiphoid view using the long axis view at $2-3 \mathrm{~cm}$ distal to the junction with the right atrium.

The maximum antero-posterior diameter and CSA in the expiratory period and minimum antero-posterior diameter and CSA in the inspiratory period were measured by loop recording and freezing the images on the monitor. Collapsibility of the diameter and collapsibility of the CSA were calculated as follows: Collapsibility of diameter $(\%)=(($ maximum diameter - minimum diameter $/$ maximum diameter $)) \times 100$. Col- 


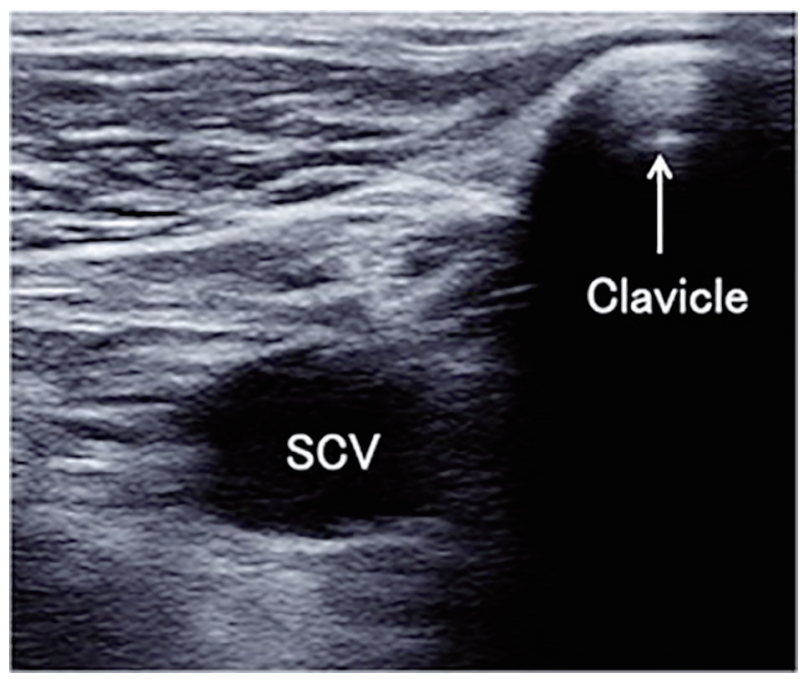

Figure 2. The ultrasound short axis view at the closest point where the subclavian vein (SCV) crosses the clavicle.

lapsibility of CSA $(\%)=(($ maximum CSA - minimum CSA $) /$ maximum CSA) $\times 100$.

All measurements were performed in the supine position. To obtain stabilized baseline values, hemodynamic and respiratory parameters were repeatedly evaluated while ultrasound examination of the veins was performed. Then, a passive leg raising maneuver (lower limbs were lifted to $40^{\circ}$ by adjusting the bed from the supine position) was performed. The reason we selected passive leg raising starting from the supine position was to minimize physical stimulation as a potential confounder. During passive leg raising, hemodynamic parameters were evaluated again; the maximum value within the first minute during passive leg raising was specified as the postpassive leg raising value. A maximum of two measurements were included for analysis if a patient needed further fluid resuscitation. As a surrogate for fluid responsiveness, a positive passive leg raising maneuver was defined as an increase in stroke volume $\geq 8 \%$ when the post-passive leg raising value was compared with the pre-passive leg raising value [8]. If the stroke volume increased $\geq 8 \%$ after passive leg raising, the patient was deemed a responder.

\section{Statistical analysis}

Patient characteristics, respiratory, hemodynamic parameters and collapsibility of veins of responders and non-responders before passive leg raising were compared using the MannWhitney U test. ROC curves of collapsibility of central veins were generated for predicting positive passive leg raising maneuver. Statistical analysis was performed with EZR version 1.30 [9].

\section{Results}

From March through October in 2014, 34 measurements in
27 patients were included in this study. Table 1 shows patient characteristics, the hemodynamic and respiratory parameters and diameters and CSA and collapsibility of each vein before passive leg raising in both responders and non-responders. Eighteen measurements fulfilled the criteria for a positive passive leg raising maneuver (responder), and 16 measurements were consistent with negative passive leg raising (nonresponder). Patient characteristics were similar except age comparing responders and non-responders. Respiratory data including tidal volume, the amount of pressure support, PEEP, or minute ventilation did not differ between the two groups, but in the responders, lower systolic and pulse pressure, higher heart rate and higher SVV were observed. Collapsibility of both the right IJV diameter and collapsibility of the CSA were greater in responders, but the collapsibility of other veins did not differ significantly comparing the two groups.

Table 2 shows the area under the ROC curves of diameters and CSAs of the central veins, and SVV before the passive leg raising measurement. The area under the ROC for collapsibility of right IJV diameter and CSA were 0.88 (95\% CI: 0.75 - 0.99) and 0.82 (95\% confidence interval (CI): 0.67 - 0.97), respectively; whereas the area under the ROC for collapsibility of the IVC diameter was 0.57 (95\% CI: $0.37-0.77)$. The area under the ROC for SVV was 0.80 (95\% CI: 0.64 - 0.96). Figure 3 shows a comparison of the ROC curve of collapsibility of the right IJV diameter and the IVC diameter. The best cut-off value of collapsibility of the right IJV to predict fluid responsiveness is $11.4 \%$ with a sensitivity of $83 \%$ and a specificity of $94 \%$.

\section{Discussion}

The main finding of this study is that the collapsibility of the antero-posterior diameter of the right IJV is a useful predictor of fluid responsiveness in hemodynamically unstable patients receiving pressure support ventilation. This is the first study to compare the predictive accuracy of fluid responsiveness among three different central veins. These results have strength in that both IJVs and both SCVs were evaluated. Previous studies examining IJVs and SCVs did not specify which side was examined [10-12].

As previous studies have shown $[1,2]$, dynamic parameters including SVV and pulse pressure variation are reliable predictors of fluid responsiveness only in patients with welladapted controlled mechanical ventilation, relatively high tidal volume $(>8 \mathrm{~mL} / \mathrm{kg})$, normal lung compliance and normal left ventricular function [13]. The passive leg raising maneuver is a more reliable method to predict fluid responsiveness in a variety of patients [14], but for interpretation of the passive leg raising maneuver, cardiac output monitoring with a pulmonary artery catheter, arterial pulse contour analysis device, continuous transthoracic or transesophageal echocardiography is needed. However, some devices are invasive, while measuring accurate cardiac output by echocardiography is associated with technical difficulties. Passive leg raising is contraindicated in some patients, due to the presence of vascular access catheters in the groin, status post-operations involving the pelvis or lower extremities, and intracranial hypertension. 
Table 1. Main Characteristics of the Responders and Non-Responders

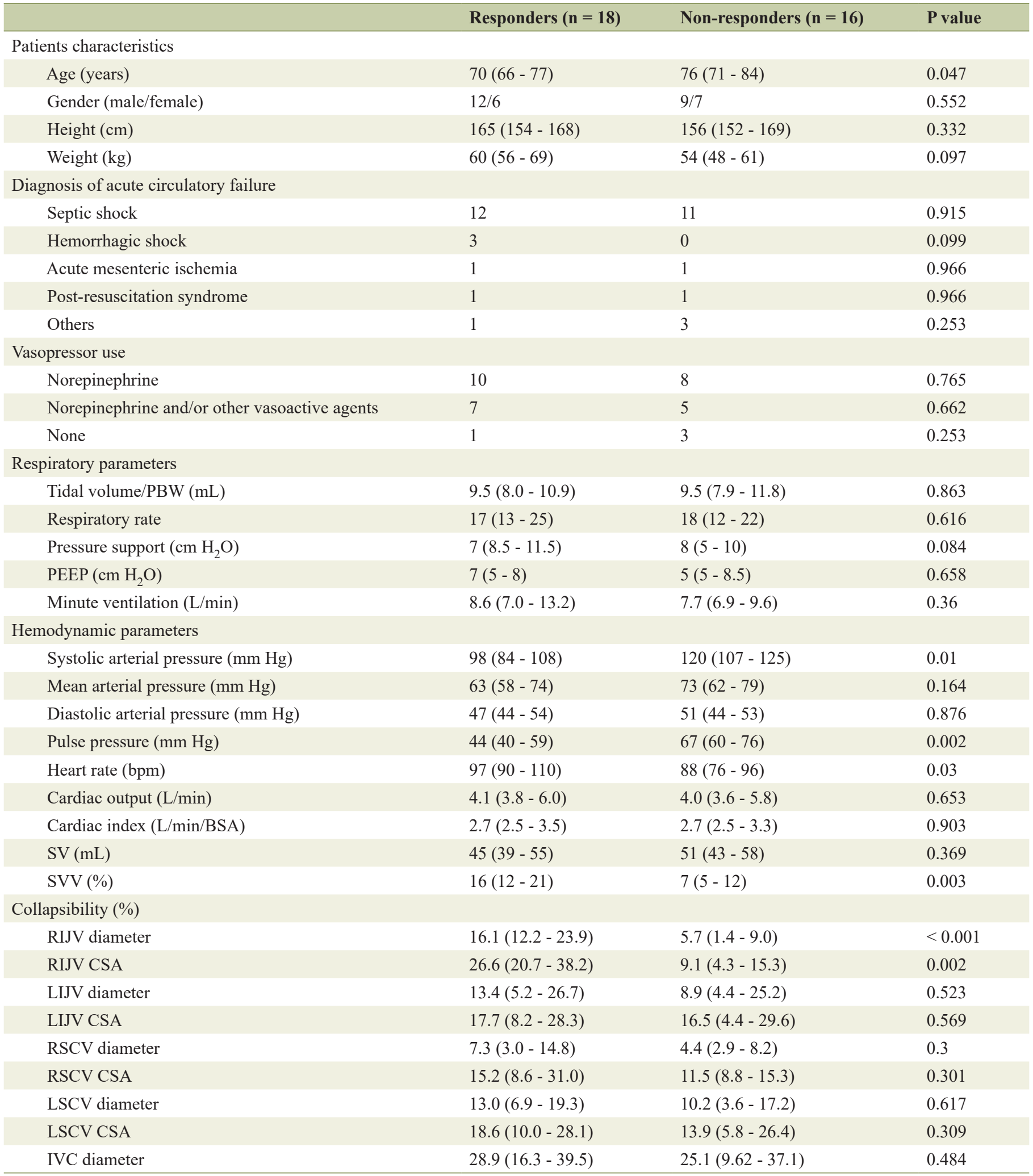

The results are expressed as the median (25-75\%). PBW: predicted body weight; PEEP: positive end expiratory pressure; BSA: body surface area; SV: stroke volume; SVV: stroke volume variation; CSA: cross-sectional area; RIJV: right internal jugular vein; LIJV: left internal jugular vein; RSCV: right subclavian vein; LSCV: left subclavian vein; IVC: inferior vena cava. 
Table 2. The Area Under the Receiver Operating Characteristic Curves $(95 \% \mathrm{Cl})$

\begin{tabular}{|c|c|}
\hline \multicolumn{2}{|l|}{ Collapsibility } \\
\hline RIJV diameter & $0.88(0.75-0.99)$ \\
\hline RIJV CSA & $0.82(0.67-0.97)$ \\
\hline LIJV diameter & $0.57(0.37-0.77)$ \\
\hline LIJV CSA & $0.56(0.35-0.77)$ \\
\hline RSCV diameter & $0.61(0.41-0.80)$ \\
\hline RSCV CSA & $0.61(0.41-0.80)$ \\
\hline LSCV diameter & $0.55(0.35-0.76)$ \\
\hline LSCV CSA & $0.60(0.40-0.81)$ \\
\hline IVC diameter & $0.57(0.37-0.77)$ \\
\hline \multicolumn{2}{|c|}{ Hemodynamic parameter } \\
\hline SVV & $0.80(0.64-0.96)$ \\
\hline
\end{tabular}

CI: confidence interval; CSA: cross-sectional area; RIJV: right internal jugular vein; LIJV: left internal jugular vein; RSCV: right subclavian vein; LSCV: left subclavian vein; IVC: inferior vena cava; SVV: stroke volume variation.

As a noninvasive dynamic parameter, respiratory changes in the IVC have been well studied with reference to central venous pressure [15], but there are a few studies examining the relationship between respiratory changes and fluid responsiveness. Several studies showed a good ability of collapsibility of the IVC to predict fluid responsiveness in patients without spontaneous breathing efforts [4, 5]; however, recent studies demonstrated collapsibility of IVC had a poorer ability to predict fluid responsiveness than expected [16-18]. In spontaneously breathing patients, respiratory changes of the IVC were less useful to predict fluid responsiveness than in patients with controlled ventilation [6]. Collapsibility of the IVC was not a good predictor of fluid responsiveness in patients with and without spontaneous breathing in recent studies, and the present results also show its poor predictive ability in patients undergoing pressure support ventilation.

Assessment of collapsibility of IJVs and SCVs appears to be easier, and therefore may be a good alternative to assessment with the IVC. However, few studies of respiratory change in SCVs and IJVs have been conducted. In a study by Kent et al, an acceptable correlation was shown between respiratory changes of SCVs and the changes of the IVC $\left(\mathrm{R}_{2}=\right.$ $0.61, \mathrm{P}<0.01)$ in patients with and without mechanical ventilation [10]. In this study, however, the ability of collapsibility of the SCV to predict fluid responsiveness was unclear. The present study shows a poor predictive ability of SCV collapsibility for responsiveness to fluid administration.

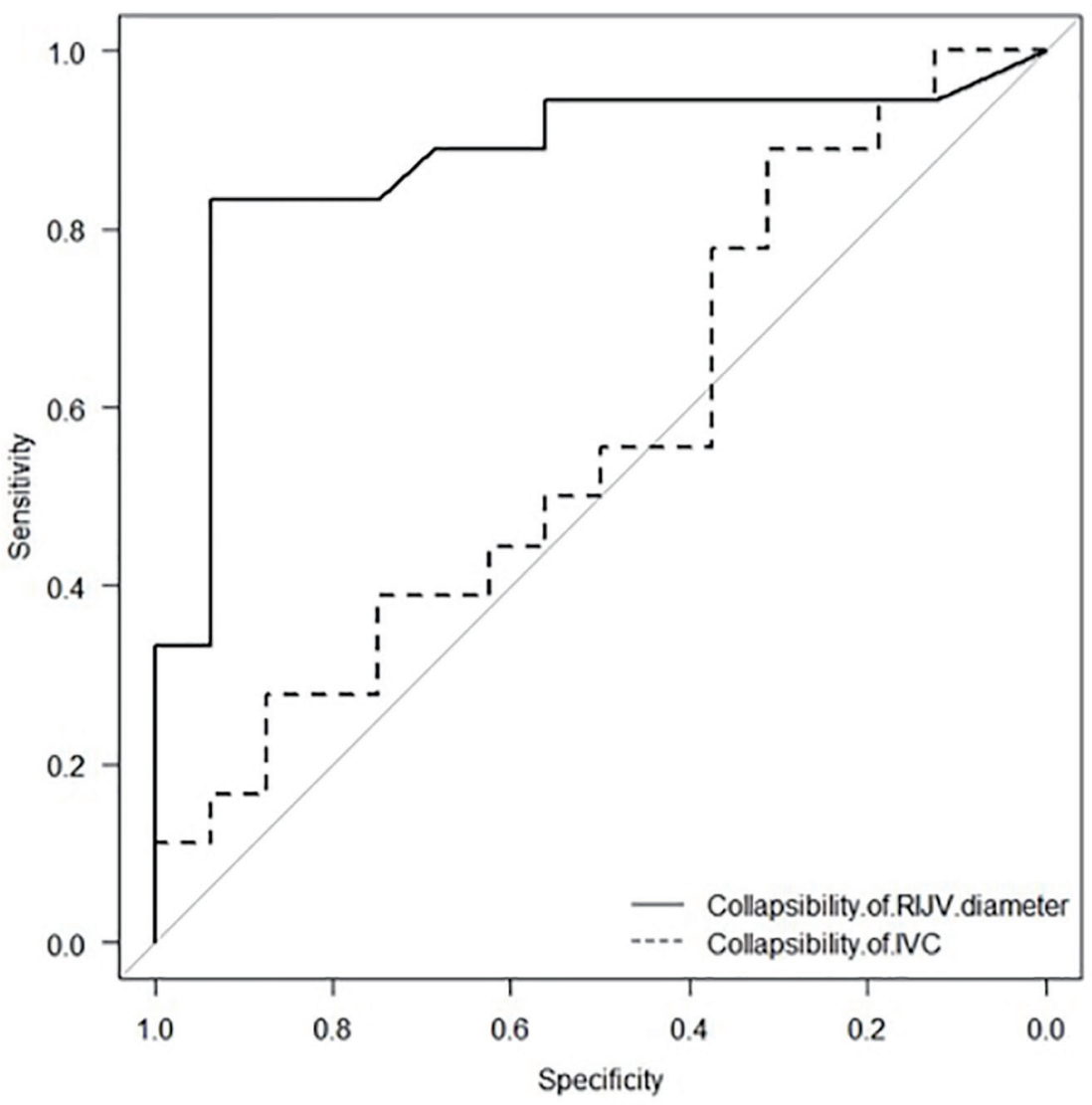

Figure 3. Receiver operating characteristic (ROC) curves of baseline collapsibility of the right internal jugular vein (RIJV) and inferior vena cava (IVC) diameters. 
An association of respiratory changes in the IVC with changes in the IJVs was also reported by Kent et al, suggesting that the correlation between the IVC and IJVs was weak [11]. In a study by Guarracino et al in 2014, respiratory change of IJV in septic patients with mandatory ventilation was highly predictive of a response to fluid administration [12]. They measured the IJV in the semi-recumbent position (head elevated $30^{\circ}$ ), and respiratory changes in the IJV calculated as the ratio of the difference in the maximal IJV antero-posterior diameter and minimum diameter to minimum diameter. They reported that a greater than $18 \%$ IJV respiratory change predicted fluid responsiveness with a sensitivity of $80 \%$ and a specificity of $95 \%$ (area under the ROC curve 0.915 , 95\% CI: $0.801-0.975$ ). Our threshold value, $11.4 \%$ of collapsibility of IJV diameter is smaller than that in the previous study [12]. This difference could be explained by the body position (semi-recumbent in [12] vs. supine position in the present study), and the different way of calculation of respiratory changes (different denominator: distensibility (the ratio of the difference in the maximum diameter and minimum diameter to minimum diameter in [12]) vs. collapsibility (the ratio of the difference in the maximum diameter and minimum diameter to maximum diameter in our study)). According to the results of [12] and the present study, respiratory changes in the IJVs could be a useful predictor of fluid responsiveness in mechanical ventilated patients regardless of the existence of spontaneous ventilation.

Also, the present results show only that collapsibility of the right IJV has a good predictive ability for responsiveness to fluid administration. The reason why the right IJV is better than the left IJV is unclear. Based on these data, the maximum diameters of the right IJV were significantly larger than the left IJV (maximum diameter: $12.6(10.7-15.1) \mathrm{mm}$ in right IJV vs. $10.2(7.2$ - 11.9) $\mathrm{mm}$ in left IJV, $\mathrm{P}<0.001)$. A possible explanation for the discrepancy is that the blood flow in the right IJV is greater than left IJV and may be affected more by the volume shift resulting from passive leg raising maneuver.

This study has several acknowledged limitations. First, we determined fluid responsiveness by stroke volume increase in arterial pulse contour analysis by passive leg raising maneuver, not by a fluid challenge. Passive leg raising is a reliable method to predict fluid responsiveness, but a fluid challenge is considered the gold standard to determine fluid responsiveness. We defined an increase in stroke volume $\geq 8 \%$ as positive passive leg raising maneuver showed in a study by Lafanechere et al [8], but they used esophageal Doppler to detect the change of stroke volume. A different way of measurement of stroke volume might affect the results of passive leg raising maneuver, although we used the third generation Flotrac ${ }^{\mathrm{TM}}$ system which is more reliable than second generation, and is comparable to semi-continuous pulmonary thermodilution method [19]. Second, the inspiratory effort was not taken into consideration in this study. Patients with strong inspiratory efforts and high tidal volume might induce greater variability of respiratory changes of the central veins. However, these results show no difference in average tidal volumes between responders and non-responders, and therefore, the influence of inspiratory effort on respiratory change in the veins could be minimal. Third, consistency of the respiratory pattern was not assured before, during or after the measurements in spontaneously breathing patients. Fourth, when generating the ROC curve, multivariate analyses including patient characteristics such as age, weight and a diagnosis of circulatory failure as covariates were not performed. Since the primary purpose of this study was to predict events, not the determination of associated factors, univariate analysis is reasonable. The results of multivariate analysis using such a small sample size may be difficult to interpret. Fifth, all measurements were performed by a single evaluator, which eliminates inter-evaluator variability. However, systematic error and bias by a single evaluator cannot be eliminated. Accuracy and validity of these results need to be confirmed in a future study. Sixth, while the study sample size (34 measurements) was not supported by a power calculation before the study, the estimated sample size is 31 measurements when we calculate an adequate sample size retrospectively using the two-sided test with a $5 \%$ level of significance and an $80 \%$ power to detect the difference between ROC curves of 0.88 (right IJV) and 0.57 (IVC). Finally, the present study excluded patients with arrhythmias, low cardiac function and acute respiratory distress syndrome. The results of this study may not be applicable to patients with these excluded conditions.

\section{Conclusion}

These results suggest that collapsibility of the right IJV diameter is a useful predictor of fluid responsiveness in patients undergoing pressure support ventilation. Collapsibility of the diameter of the IVC, left IJV and bilateral SCVs are not good predictors of fluid responsiveness in these patients. Further studies are needed to validate the ability of collapsibility of the right IJV to predict responsiveness to fluid administration in patients under various assisted modes of ventilation who require fluid resuscitation.

\section{Acknowledgments}

None to declare.

\section{Financial Disclosure}

None to declare.

\section{Conflict of Interest}

The authors declare that there is no conflict of interest.

\section{Informed Consent}

Due to the noninvasive nature of the study, the Institutional Review Board waived the need for informed consent of enrolled patients. 


\section{Author Contributions}

YI, TN and MS designed the study; YI collected the data and drafted the manuscript; TN, MS, YM, AA and AL helped to interpret the data and to revise the manuscript. All authors also read and approved the final manuscript.

\section{Data Availability}

The data supporting the findings of this study are available from the corresponding author upon reasonable request.

\section{References}

1. Marik PE, Baram M, Vahid B. Does central venous pressure predict fluid responsiveness? A systematic review of the literature and the tale of seven mares. Chest. 2008;134(1):172-178.

2. Osman D, Ridel C, Ray P, Monnet X, Anguel N, Richard C, Teboul JL. Cardiac filling pressures are not appropriate to predict hemodynamic response to volume challenge. Crit Care Med. 2007;35(1):64-68.

3. Marik PE, Monnet X, Teboul JL. Hemodynamic parameters to guide fluid therapy. Ann Intensive Care. 2011;1(1):1.

4. Barbier C, Loubieres Y, Schmit C, Hayon J, Ricome JL, Jardin F, Vieillard-Baron A. Respiratory changes in inferior vena cava diameter are helpful in predicting fluid responsiveness in ventilated septic patients. Intensive Care Med. 2004;30(9):1740-1746.

5. Feissel M, Michard F, Faller JP, Teboul JL. The respiratory variation in inferior vena cava diameter as a guide to fluid therapy. Intensive Care Med. 2004;30(9):18341837.

6. Muller L, Bobbia X, Toumi M, Louart G, Molinari N, Ragonnet B, Quintard H, et al. Respiratory variations of inferior vena cava diameter to predict fluid responsiveness in spontaneously breathing patients with acute circulatory failure: need for a cautious use. Crit Care. 2012;16(5):R188.

7. Uthoff H, Siegemund M, Aschwanden M, Hunziker L, Fabbro T, Baumann U, Jaeger KA, et al. Prospective comparison of noninvasive, bedside ultrasound methods for assessing central venous pressure. Ultraschall Med. 2012;33(7):E256-E262.

8. Lafanechere A, Pene F, Goulenok C, Delahaye A, Mallet V, Choukroun G, Chiche JD, et al. Changes in aor- tic blood flow induced by passive leg raising predict fluid responsiveness in critically ill patients. Crit Care. 2006;10(5):R132.

9. Kanda Y. Investigation of the freely available easy-touse software 'EZR' for medical statistics. Bone Marrow Transplant. 2013;48(3):452-458.

10. Kent A, Bahner DP, Boulger CT, Eiferman DS, Adkins EJ, Evans DC, Springer AN, et al. Sonographic evaluation of intravascular volume status in the surgical intensive care unit: a prospective comparison of subclavian vein and inferior vena cava collapsibility index. J Surg Res. 2013;184(1):561-566.

11. Kent A, Patil P, Davila V, Bailey JK, Jones C, Evans DC, Boulger CT, et al. Sonographic evaluation of intravascular volume status: Can internal jugular or femoral vein collapsibility be used in the absence of IVC visualization? Ann Thorac Med. 2015;10(1):44-49.

12. Guarracino F, Ferro B, Forfori F, Bertini P, Magliacano L, Pinsky MR. Jugular vein distensibility predicts fluid responsiveness in septic patients. Crit Care. 2014;18(6):647.

13. Pinsky MR. Understanding preload reserve using functional hemodynamic monitoring. Intensive Care Med. 2015;41(8):1480-1482.

14. Cavallaro F, Sandroni C, Marano C, La Torre G, Mannocci A, De Waure C, Bello G, et al. Diagnostic accuracy of passive leg raising for prediction of fluid responsiveness in adults: systematic review and meta-analysis of clinical studies. Intensive Care Med. 2010;36(9):1475-1483.

15. Beigel R, Cercek B, Luo H, Siegel RJ. Noninvasive evaluation of right atrial pressure. J Am Soc Echocardiogr. 2013;26(9):1033-1042.

16. Theerawit $P$, Morasert $T$, Sutherasan $Y$. Inferior vena cava diameter variation compared with pulse pressure variation as predictors of fluid responsiveness in patients with sepsis. J Crit Care. 2016;36:246-251.

17. de Oliveira OH, Freitas FG, Ladeira RT, Fischer CH, Bafi AT, Azevedo LC, Machado FR. Comparison between respiratory changes in the inferior vena cava diameter and pulse pressure variation to predict fluid responsiveness in postoperative patients. J Crit Care. 2016;34:46-49.

18. Charbonneau H, Riu B, Faron M, Mari A, Kurrek MM, Ruiz J, Geeraerts T, et al. Predicting preload responsiveness using simultaneous recordings of inferior and superior vena cavae diameters. Crit Care. 2014;18(5):473.

19. De Backer D, Marx G, Tan A, Junker C, Van Nuffelen M, Huter L, Ching W, et al. Arterial pressure-based cardiac output monitoring: a multicenter validation of the thirdgeneration software in septic patients. Intensive Care Med. 2011;37(2):233-240. 